\title{
ON THE NONEXISTENCE OF SOLUTIONS OF DIFFERENTIAL EQUATIONS IN NONREFLEXIVE SPACES ${ }^{1}$
}

\author{
BY ARRIGO CELLINA
}

Communicated by M. H. Protter, May 8, 1972

We consider the problem of the existence of solutions of ordinary differential equations in Banach spaces. Counterexamples in $c_{0}$ by Dieudonné [2] and in $l_{2}$ by Yorke [4] show that, in infinite-dimensional spaces, Peano's existence theorem need not necessarily be true. A natural question then is that of asking whether there could exist infinite-dimensional Banach spaces on which Peano's theorem holds, or otherwise, whether the truth of Peano's theorem is a characterization of the finite dimensionality.

This paper offers only a partial answer to this question: Our Theorem 1 below states that there exist no nonreflexive spaces on which Peano's theorem holds.

THEOREM 1. Let $X$ be a nonreflexive Banach space. Then there exists a continuous $F: R \times X \rightarrow X$ such that the Cauchy problem

$$
x^{\prime}=F(t, x), \quad x(0)=0,
$$

admits no solution on any nonvanishing interval $[a, b]$ containing the origin.

Proof. Call $B$ the unit ball of $X$. From R. C. James' characterization of reflexivity [3] it follows that there exists a $v \in X^{*},\|v\|=1$, such that, for every $x \in B,\langle v, x\rangle<1$. We shall now construct a fixed-point-free continuous mapping $f$ of $B$ into itself with some special properties.

By definition of norm of $v$, we can recursively define a sequence of points $x_{n},\left\|x_{n}\right\|=1$, such that $\left\langle v, x_{n}\right\rangle\left\langle\left\langle v, x_{n+1}\right\rangle\right.$ and $\left\langle v, x_{n}\right\rangle \rightarrow 1$, and consider the sets

$$
\begin{gathered}
O_{1}=\left\{x \in B:\langle v, x\rangle<2\left\langle v, x_{2}\right\rangle-1\right\}, \\
O_{n}=\left\{x \in B: 2\left\langle v, x_{n-1}\right\rangle-1<\langle v, x\rangle<2\left\langle v, x_{n+1}\right\rangle-1\right\} .
\end{gathered}
$$

Then every $O_{j}$ is open (relative to $B$ ) and their union covers $B$. Moreover it is not difficult to check that every point $x \in B$ belongs to at most two $O_{j}$ and has a neighborhood that meets at most three $O_{j}$, so that the covering $\left\{O_{n}\right\}$ is locally finite and has a partition of unity subordinated

AMS 1970 subject classifications. Primary 34A10, 34G05; Secondary 46B10.

Key words and phrases. Ordinary differential equations, Cauchy problem, nonexistence of solutions, nonreflexive spaces.

${ }^{1}$ Supported in part by the CNR, Comitato per la Matematica. 
to it. Call $p_{n}$ this partition and define $f$ to be

$$
f(x)=\frac{1}{2} \sum_{i=1}^{\infty} p_{i}(x)\left(\left\langle v, x_{i+1}\right\rangle\right)^{-1}(\langle v, x\rangle+1) x_{i+1} .
$$

Then $f$ is a continuous map $B \rightarrow X$. Moreover fix $x \in B$ and let $\hat{n}$ be such that $x$ belongs at most to $O_{\hat{n}}$ and $O_{n+1}$. Then

$$
\begin{aligned}
\|f(x)\| & \leqq \frac{1}{2}\left\{p_{\hat{n}}(x)\left(\left\langle v, x_{\hat{n}+1}\right\rangle\right)^{-1}+p_{\hat{n}+1}(x)\left(\left\langle v, x_{\hat{n}+2}\right\rangle\right)^{-1}\right\}(\langle v, x\rangle+1) \\
& <\frac{1}{2}\left\{p_{\hat{n}}(x)\left(\left\langle v, x_{\hat{n}+1}\right\rangle\right)^{-1} 2\left\langle v, x_{\hat{n}+1}\right\rangle\right\}+\frac{1}{2}\left\{p_{\hat{n}+1}(x)\left(\left\langle v, x_{\hat{n}+2}\right\rangle\right)^{-1}\right. \\
& \left.\quad \cdot 2\left\langle v, x_{\hat{n}+2}\right\rangle\right\} \\
& =1 .
\end{aligned}
$$

Therefore $f: B \rightarrow B$. In addition we have that

$$
\langle v, f(x)\rangle=\frac{1}{2} \sum_{i=1}^{\infty} p_{i}(x)(\langle v, x\rangle+1)=\frac{1}{2}(\langle v, x\rangle+1) .
$$

This last equation implies the nonexistence of fixed points of $f$. In fact if $f(\xi)=\xi$, we would have $\langle v, \xi\rangle=1$, contradicting our choice of $v$.

Let $\mathscr{F}: X \rightarrow B$ be an extension of $f$ to the whole $X$, with range in $B$, and define the function $F: R \times X \rightarrow X$ by

$$
\begin{aligned}
F(t, x) & =2 t \mathscr{F}\left(x / t^{2}\right), & & t \neq 0, \\
& =0, & & t=0 .
\end{aligned}
$$

Since $\|\mathscr{F}\| \leqq 1$, it follows that $F$ is continuous on $R \times X$. Consider the Cauchy problem (CP) with the above defined $F$ and let $y:[a, b] \rightarrow X$ be a solution, where $0 \in[a, b]$. For any given $t \in[a, b]$,

$$
\begin{aligned}
\|y(t)\| & \leqq\left|\int_{0}^{t}\|F(s, y(s))\| d s\right| \\
& \leqq\left|\int_{0}^{t} 2 s d s\right|=t^{2}
\end{aligned}
$$

so that $\left\|y / t^{2}\right\| \leqq 1$.

Hence, along such a solution, $F(t, y)=2 t f\left(y / t^{2}\right)$. Moreover we have

$$
\begin{aligned}
\langle v, y\rangle^{\prime} & =\left\langle v, y^{\prime}\right\rangle=2 t\left\langle v, f\left(y / t^{2}\right)\right\rangle \\
& =2 t(1 / 2)\left(\left\langle v, y / t^{2}\right\rangle+1\right)=(1 / t)\langle v, y\rangle+t .
\end{aligned}
$$

The only solution of $\xi^{\prime}=(1 / t) \xi+t, \quad \xi(0)=0$, satisfying $|\xi| \leqq t^{2}$ is $\xi(t)=t^{2}$, so that $\langle v, y(t)\rangle=t^{2}$ for $t \in[a, b]$ or $\left\langle v, y(t) / t^{2}\right\rangle=1$. Since $\left\|y(t) / t^{2}\right\| \leqq 1$, this contradicts our assumptions on $v$.

The technique used in the proof of the above theorem, of taking a 
special fixed-point-free self mapping $f$ of $B$, extending it to $\mathscr{F}$ and defining $F(t, x)$ as $2 t \mathscr{F}\left(x / t^{2}\right)$ can be used to give explicit examples of differential equations without existence in $l_{1}$ and $l_{\infty}$, which are noteworthy because of their simplicity.

In $l_{\infty}$ consider the mapping $f$ given by

$$
f\left(x_{1}, x_{2}, \ldots, x_{n}, \ldots\right)=\left(1-\|x\|,\left|x_{1}\right|^{1 / 2}, \ldots,\left|x_{n-1}\right|^{1 / 2}, \ldots\right) .
$$

This is a continuous fixed-point-free mapping $B \rightarrow B$. Extend it to a $\mathscr{F}: X \rightarrow B$ and define $F$ as before. It follows again that for a possible solution $y(t)$ we have $\left\|y(t) / t^{2}\right\| \leqq 1$ so that the following system has to hold:

$$
\begin{aligned}
y_{1}^{\prime} & =2 t\left(1-\left\|y / t^{2}\right\|\right), \\
y_{n+1}^{\prime} & =2\left|y_{n}\right|^{1 / 2}, \quad n=1,2, \ldots .
\end{aligned}
$$

We see that every $y_{i}$ is nonnegative and that $y_{1}$ cannot be identically zero on any interval $[0, \delta]$ since then, on one hand, every $y_{n}$ would be identically zero on that interval, while on the other, for every $t,\|y\|=$ $\sup \left\{y_{1}(t), \ldots, y_{n}(t), \ldots\right\}$ should be $t^{2}$. So $0=\inf \left\{t>0: y_{1}(t)>0\right\}$.

Equations (2) are the equations of the process of successive approximations for the problem $x^{\prime}=2|x|^{1 / 2}, x(0)=0$, with first approximation $y_{1}$. By a result of Dieudonné described in [1, p. 427], this process converges to the solution $x(t)=t^{2}$. Then on $[0, \delta],\|y\|=t^{2}$ or $y_{1}^{\prime} \equiv 0$. This implies $0 \equiv y_{1} \equiv y_{2} \equiv \ldots \equiv y_{n} \equiv \ldots$ on $[0, \delta]$ contradicting $\|y\|=t^{2}$.

In $l_{1}$ consider the mapping $f$ defined by

$$
f\left(x_{1}, x_{2}, \ldots, x_{n}, \ldots\right)=\left(1-\|x\|, x_{1}, \ldots, x_{n-1}, \ldots\right) .
$$

It can be checked that this is a continuous fixed-point-free mapping of $B$ into itself. Extending $f$ and defining a continuous $F(t, x)$ as before, we see again that a possible solution $y$ of $(\mathrm{CP})$ has to be such that $\|y(t)\| \leqq t^{2}$ and has to satisfy, on any interval $[0, \delta]$, the system

$$
\begin{aligned}
y_{1}^{\prime} & =2 t\left(1-\left\|y / t^{2}\right\|\right), \\
y_{n+1}^{\prime} & =2 y_{n} / t, \quad n=1,2, \ldots .
\end{aligned}
$$

Then every $y_{i}^{\prime}$ and every $y_{i}$ is nonnegative. Moreover we have

$$
\begin{aligned}
\|y(t)\| & =\sum_{i=1}^{\infty} y_{i}(t)=\sum_{i=1}^{\infty} \int_{0}^{t} y_{i}^{\prime}(s) d s \\
& =\int_{0}^{t} 2 s\left(1-\|y\| / s^{2}\right) d s+2 \sum_{i=1}^{\infty} \int_{0}^{t} y_{i}(s) / s d s .
\end{aligned}
$$

Since the sequence $g_{n}(s)=\sum_{i=1}^{n} y_{i}(s) / s$ is nondecreasing and bounded 
above by the integrable function $\|y(s) / s\|$,

$$
\sum_{i=1}^{\infty} \int_{0}^{t} y_{i}(s) / s d s=\lim _{n \rightarrow \infty} \int_{0}^{t} g_{n}(s) d s=\int_{0}^{t}\|y(s) / s\| d s .
$$

Hence from (3) we have, for every $t \in[0, \delta]$,

$$
\begin{aligned}
\|y(t)\| & =\int_{0}^{t} 2 s(1-\|y / s\|) d s+\int_{0}^{t} 2\|y / s\| d s \\
& =\int_{0}^{t} 2 s \quad d s=t^{2} .
\end{aligned}
$$

This implies $y_{1}^{\prime} \equiv 0$ and consequently $0 \equiv y_{1} \equiv y_{2} \equiv \ldots \equiv y_{n} \equiv \ldots$. This contradicts $\|y\|=t^{2}$. Therefore no such solution $y$ can exist.

\section{REFERENCES}

1. F. Brauer and S. Sternberg, Local uniqueness, existence in the large, and the convergence of successive approximations, Amer. J. Math. 80 (1958), 421-430. MR 20 \# 1806.

2. J. Dieudonné, Deux exemples singuliérs d'équations différentielles, Acta Sci. Math. Szeged 12 (1950), Leopoldo Fejér et Frederico Riesz LXX annos natis dedicatus, pars B, 38-40. MR 11, 729.

3. R. C. James, Characterizations of reflexivity, Studia Math. 23 (1963/64), 205-216. MR 30 \# 431.

4. J. Yorke, A continuous differential equation in Hilbert space without existence, Funkcial. Ekvac. 13 (1970), 19-21. MR 41 \#8792.

Istituto di Matematica, Viale Morgagni 67/A, I 50134 Firenze, Italy 